
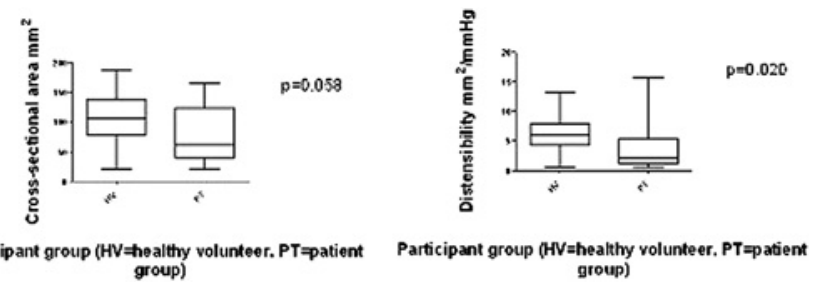

Abstract PTU-178 Figure 1 Cross-sectional area and distensibility at $30 \mathrm{ml}$ EndoFLIP balloon volume in healthy volunteer and patient groups.

Conclusion EndoFLIP ${ }^{\circledR}$ is not useful for GERD diagnosis as EGJ CSA and distensibilty do not distinguish between $\mathrm{HVs}$ and GERD patients defined by clinical presentation or $\mathrm{pH}$ measurement. This unexpected result may be due to an important interaction of obesity with EndoFLIP ${ }^{\circledR}$ measurements.

Competing interests None declared.

\section{PTU-179 ACETOWHITENING AS A NOVEL DIAGNOSTIC TOOL FOR THE DIAGNOSIS AND CHARACTERISATION OF NEOPLASIA WITHIN BARRETT'S OESOPHAGUS}

doi:10.1136/gutjnl-2012-302514c.179

G Longcroft-Wheaton, ${ }^{*}$ P Bhandari. Gastroenterology, Portsmouth Hospitals NHS Trust, Portsmouth, UK

Introduction The identification of Barrett's neoplasia using acetic acid has relied upon the examination of surface patterns, which involves subjective judgement. When sprayed onto Barrett's tissue acetic acid causes an acetowhitening reaction. We have observed that neoplastic tissue loses this whitening more quickly than nonneoplastic Barrett's. This study aims to quantify the acetowhitening time and develop an objective tool for the diagnosis of Barrett's neoplasia.

Methods Patients referred for suspected Barrett's neoplasia and for routine Barrett's surveillance were assessed. A $50 \mathrm{ml}$ mucolytic drink of $10 \% \mathrm{~N}$-acetyl cysteine and $5 \mathrm{ml}$ of simethicone was taken prior to the procedure. Fujinon gastroscopes with EPX 4400 processor were used. $20 \mathrm{ml}$ of $2.5 \%$ acetic acid was applied to the Barrett's mucosa via a spray catheter. Timing was recorded with a stopwatch and started after the oesophagus had been coated with acetic acid and the excess dye sucked away. Disappearance of the acetowhitening was defined as the appearance of erythema within the Barrett's epithelium. After a maximum of 5 min the Barrett's epithelium was washed using $20 \mathrm{ml}$ of water and targeted biopsies of any neoplasia and quadrantic $2 \mathrm{~cm}$ biopsies of the residual Barrett's was taken. The histology was correlated to the acetowhitening disappearance time. ROC curves were produced to identify threshold timings for optimum sensitivity and specificity for high risk neoplasia and invasive cancer within Barrett's.

Results Data from 146 areas of Barrett's was collected from 121 patients. 84\% were male. Mean age 69. 72/86 metaplasia, 6/14 LGD, 26/27 HGD, 15/15 IMC and 12/12 areas of invasive cancer were recognised correctly by the endoscopist. A ROC curve was produced for the identification of high risk neoplasia (HGD, IMC and invasive cancer) using the acetowhitening timings. The area under the curve was 0.93 (95\% CI 0.89 to 0.97 ), with an asymptomatic significance of $\mathrm{p}=0.000$. Using a threshold of $142 \mathrm{~s}$ a sensitivity for neoplasia of $98 \%(95 \%$ CI $89 \%$ to $100 \%)$ and specificity of $84 \%$ (95\% CI $74 \%$ to $91 \%$ ) was achieved. A further ROC curve was produced for HGD + IMC vs invasive cancer. The area under the curve was 0.786 (61 to 96). Using a cut off of $20 \mathrm{~s}$, a sensitivity for invasive cancer of $67 \%$ ( $95 \%$ CI $35 \%$ to $90 \%$ ) and specificity of $85 \%$ (95\% CI $69 \%$ to $95 \%)$ could be achieved.
Conclusion The acetowhitening reaction can be exploited for the diagnosis of neoplasia within Barrett's. This provides the endoscopist with an objective and simple numerical tool for predicting whether an area is neoplastic, and to predict the presence of submucosally invasive disease. This has important implications for the treatment of neoplasia within Barrett's, and could prevent inappropriate attempts at endoscopic resection of invasive cancer.

Competing interests None declared.

\section{PTU-180 WHAT IS THE ROLE OF EUS IN NODAL STAGING OF OESOPHAGEAL CANCER IN THE ERA OF PET-CT?}

doi:10.1136/gutjnl-2012-302514c.180

${ }^{1} \mathrm{G} J$ Bryce, ${ }^{*}{ }^{1} \mathrm{M} J$ Forshaw, ${ }^{2} \mathrm{~A} J$ Stanley, ${ }^{2} \mathrm{~N}$ Jamieson, ${ }^{3} \mathrm{~S}$ Paterson, ${ }^{4} \mathrm{~S}$ Ballantyne. ${ }^{1}$ Department of UGI Surgery, Glasgow Royal Infirmary, Glasgow, UK; ${ }^{2}$ Department of Gastroenterology, Glasgow Royal Infirmary, Glasgow, UK; ${ }^{3}$ Department of Gastroenterology, Stirling Royal Infirmary, Stirling, UK; ${ }^{4}$ Department of Radiology, Gartnavel General Hospital, Glasgow, UK

Introduction EUS+/-FNA has been regarded as a standard investigation for $\mathrm{T}$ and $\mathrm{N}$ staging of oesophageal and oesophagogastric junctional (OGJ) cancer. The increased availablility of PET-CT has led to many centres reducing their use of EUS and relying more on non-invasive assessment of lymph node involvement. The aim of this study was to retrospectively analyse the outcomes from EUS following the introduction of PET-CT into a single regional unit.

Methods The computerised records of all patients diagnosed with oesophageal or OGJ cancer and discussed at a regional MDM between March 2009 and February 2011 were analysed. Patients felt to be suitable for radical treatment based upon initial endoscopy, CT scan and review of referral letter underwent a combination of PETCT +/- EUS. The final staging pathway and management of this group of patients were analysed retrospectively.

Results 593 patients were diagnosed and presented to the regional MDM. 412 (69\%) were directed towards palliative treatment following initial assessment. Of the remaining 181 (31\%), PET-CT was undertaken in 180 and EUS in 99 (55\%). FNA was undertaken in $31(31 \%)$ of those undergoing EUS. One patient $(1 \%)$ had a perforation related to dilatation prior to planned EUS. A covered stent was inserted and he was discharged from hospital. The findings on PET-CT directly changed management to a palliative approach in 30 patients (17\%). A further 75 patients (42\%) required further investigations based upon PET-CT including EUS $(n=52)$, colonoscopy $(n=9)$, review by other specialities $(n=6)$, lymph node excision biopsy $(n=2)$, radiological guided FNA/core biopsy $(n=3)$ and MRI liver $(n=2)$. EUS was performed to investigate nodal status in $51(52 \%)$, to confirm the presence or depth of tumour invasion in $21(21 \%)$ and to investigate other organ involvement in $3(3 \%) .24$ EUS procedures were performed routinely due to protocols used at that time. EUS+/-FNA directed patients to a palliative approach in $22(22 \%)$. Management was directed to a radical approach in 72 $(73 \%)$, and to endoscopic treatment (EMR/PDT) in 5 (5\%). In the 98 patients who had both PET-CT and EUS, there was concordance of lymph node status in 79 (85\%). Ten $(11 \%)$ patients with negative nodes on PET-CT had positive nodes on EUS (of which 5 were suspicious at the time of staging CT), and 4 (4\%) with positive nodes on PET-CT had negative nodes on EUS. Five had incomplete EUS due to stricturing.

Conclusion These results demonstrate that EUS has a complementary role in the staging process, with EUS playing an essential role in $11 \%$ of patients where confirmation of lymph node status, not identified on PET-CT, guided appropriate management.

Competing interests None declared. 